\title{
HACIA LA CONVERGENCIA DE LOS SISTEMAS DE EDUCACIÓN SUPERIOR EN AMÉRICA LATINA
}

\author{
Norberto Fernández Lamarra $(*)$
}

SÍNTESIS: La convergencia y la articulación de los sistemas de educación superior en América Latina, y la construcción y consolidación del Espacio Latinoamericano de Educación Superior, se plantean en este artículo como fuertes desafíos y como procesos ineludibles frente a la actual situación de fragmentación, de diversificación institucional y de disparidad en los niveles de calidad, en especial a partir de los años 90.

Por todo lo expuesto, se realiza una caracterización de la situación actual y de la evolución reciente de la educación superior en América Latina, planteando los avances registrados en materia de evaluación y acreditación de la calidad, y reseñando los principales programas de convergencia desarrollados en el marco de los procesos de integración regional. Además, se analizan el Mecanismo Experimental de Acreditación de Carreras del MERCOSUR (MEXA); los programas, proyectos, trabajos y estudios del IESALC de la UNESCO, de la OEI, de la RIACES, de la Comisión Europea, y de las diversas redes de universidades existentes. Entre ellas se consideran Columbus, el Grupo Montevideo, el CSUCA, el CINDA, la Red Latinoamericana de Cooperación Universitaria y la Asociación de Universidades de América Latina y del Caribe para la Integración. Finalmente, se plantean algunas propuestas para responder al desafío de consolidar el Espacio Latinoamericano de Educación Superior y su convergencia con el Espacio Europeo, en especial con el Proceso de Bolonia.

SÍNTESE: A convergência e a articulação dos sistemas de educação superior na América Latina, e a construção e a consolidação do Espaço Latino-americano de Educação Superior são apresentadas, neste artigo, como fortes desafios e como processos inevitáveis frente à atual situação de fragmentação, de diversificação institucional e de disparidade nos níveis de qualidade, especialmente a partir dos anos 90 .

Com esta exposição, caracteriza-sea situação atual e a evolução recente da educação superior na América Latina, apresentam-se os avanços registrados em matéria de avaliação e acreditação da qualidade, e

(*) Vicerrector académico, a cargo del Rectorado, del Instituto Universitario ISALUD, y catedrático de Administración de la Educación en las universidades nacionales de Buenos Aires, La Plata y Tres de Febrero, Argentina. 
resenham-se os principais programas de convergência desenvolvidos no marco dos processos de integração regional. Ademais, analisam-se o Mecanismo Experimental de Acreditação de estudos de graduação do MERCOSUL (MEXA); OS programas, projetos, trabalhos e estudos do IESALC da UNESCO, da OEI, da RIACES, da Comissão Européia, e das diversas redes de universidades existentes. Entre elas estão consideradas Columbus, Grupo Montevidéu, CSUCA, CINDA, a Rede Latino-americana de Cooperação Universitária e a Associação de Universidades da América Latina e do Caribe para a Integração. Finalmente, apresentam-se algumas propostas para responder ao desafio de consolidar o Espaço Latino-americano de Educação Superior e sua convergência com o Espaço Europeu, especialmente com o Processo de Bolonha.

\section{EVOLUCIÓN DE LA EDUCACIÓN SUPERIOR EN AMÉRICA LATINA}

Los sistemas de educación superior han registrado en las dos últimas décadas un proceso de fuerte diversificación, tanto en su organización como en su calidad, con la inclusión de modelos universitarios diferentes y contradictorios. Tal situación es muy diferente a la que había existido hasta la década de los 80 , pues la educación superior había sido casi siempre estatal y con fuerte autonomía institucional y académica a partir del Movimiento de la Reforma Universitaria originado en la Universidad Nacional de Córdoba en el año 1918. El modelo predominante era el napoleónico, en el que las facultades de carácter profesional se constituían en el centro de la organización universitaria. En ese modelo las carreras son largas, de tipo túnel -como las denominan los europeos-, en las que los alumnos obtienen el título después de seis o siete años de estudios, y en las que no existen ciclos ni títulaciones intermedios.

A lo largo de buena parte del siglo Xx el modelo napoleónico de universidad y las concepciones de autonomía universitaria y de primacía de la universidad pública se fueron afianzando en la mayor parte de los países latinoamericanos. El número de instituciones universitarias - por lo general estatales- fue creciendo poco a poco, y los niveles de calidad se mantuvieron más o menos homogéneos hasta la década de los 80 .

En los años finales de esa década y en los inicios de la de los 90 se introdujeron en el marco de los procesos de globalización estrategias de carácter neoliberal que tendieron a reemplazar las políticas de bienestar impulsadas por el Estado, por otras en las que predominaron las concepciones de mercado y de privatización de los servicios públicos, entre ellos la educación. Las crisis nacionales en materia económica 
Ilevaron a una fuerte restricción del financiamiento público para los sectores sociales en general, y para la educación y la universidad en particular.

A pesar de esto creció la demanda de educación superior por parte de la población, incrementándose en gran medida las tasas de escolarización y el número de estudiantes. Para atender dicha demanda se crearon diversos tipos de instituciones de educación superior universitarias y no universitarias -en su mayoría de carácter privado-, y sin criterios previos en cuanto a niveles de calidad y de pertinencia institucional. La educación superior en América Latina registró incrementos a partir de la segunda mitad del siglo XX. El número de instituciones universitarias pasó de 75, en 1950, a más de 1.500 en la actualidad, que en su mayoría son privadas. El número de estudiantes pasó de 267.000 , en 1950 , a casi 12 millones, es decir, que la matrícula se multiplicó en 50 años 45 veces.

\section{CUADRO 1}

Instituciones universitarias en América Latina

$1950 \longrightarrow 75$
$1975 \longrightarrow 330$
$1985 \longrightarrow 450$
$1995 \longrightarrow 812$ (319 públicas y 493 privadas)
$2003 \longrightarrow$ Más de 1.500

\section{CUADRO 2}

Número de estudiantes de educación superior en América Latina

$1950 \longrightarrow 267.000$
$1970 \longrightarrow 1.640 .000$
$1980 \longrightarrow 4.930 .000$
$1990 \longrightarrow 7.350 .000$
$2000 \longrightarrow 2.000 .000$ apróx.


La tasa de incremento anual de la matrícula, desde 1990, ha sido del $6 \%$, y mucho mayor para la universidad privada (8\%) que para la pública $(2,5 \%)$ Eso ha llevado a que hoy más del $50 \%$ de la matrícula universitaria en la región concurra a universidades privadas, a diferencia de lo que ocurría hasta la década de los 80 , en los que la universidad latinoamericana era sobre todo pública, tal como ya fue señalado.

El crecimiento de la matrícula ha llevado a un incremento importante de la tasa bruta de escolarización terciaria: del $2 \%$ en 1950 , al 19\% en 2000 (se multiplicó por 10 en 50 años). Sin embargo, esta tasa es muy inferior a la de los países desarrollados: 51,6\% en 1997.

CUADRO 3

Tasa bruta de escolarización terciaria en América Latina

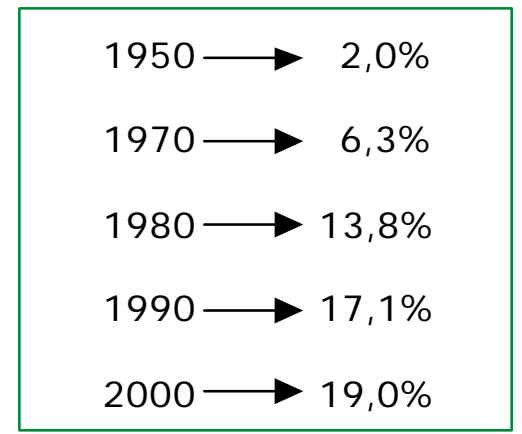

En síntesis, se registró un fuerte crecimiento de la matrícula y del número de instituciones de educación superior, una clara tendencia al incremento de las instituciones privadas, una gran diversificación institucional, y una alta disparidad en los niveles de calidad.

J unto con la diversificación institucional y con la disparidad en relación con la calidad, aparece una gran heterogeneidad en materia de modelos universitarios, que se constituyen más como «deformaciones» del modelo napoleónico que como superadores del mismo. Se podría plantear que, en muchos casos, se mantienen y se profundizan ciertos aspectos negativos del modelo profesionalista por facultades, y se introducen algunos de los aspectos menos deseables de los múltiples modelos vigentes en Estados Unidos. Así, se crean en muchos países de América Latina instituciones universitarias privadas que son denominadas «universidades garajes», por sus dimensiones, por su baja calidad y 
por el tipo de infraestructura física disponible. En algunos países es difícil actualizar la información sobre el número de instituciones de educación superior existentes por la falta de registros adecuados. EI propio Instituto Internacional para la Educación Superior en América Latina y el Caribe (IESALC) de la UNESCO no ha podido disponer hasta ahora de información regional precisa sobre el número de instituciones existentes de este nivel.

A esta caótica heterogeneidad institucional se agrega la gran disparidad en materia de planes de estudio, con denominación de titulaciones diversa, con objetivos formativos también diferentes, y, por ende, con duración de los estudios muy disímiles. Así, se encuentran en un mismo país designaciones desiguales de las titulaciones de una misma área profesional, y, a su vez, duraciones igualmente distintas de una misma carrera ${ }^{1}$.

Frente a este panorama reseñado brevemente, en América Latina surgen con énfasis los procesos de aseguramiento de la calidad a través de la evaluación de las instituciones y de la acreditación de carreras de grado y de postgrado, a los que se hará referencia más adelante ${ }^{2}$.

\subsection{EL DESARROLLO DE LOS POSTGRADOS}

Durante la década de los 90 se registró en toda América Latina, y con mayor intensidad en al gunos países -entre ellos Argentina, Brasil, Chile y Colombia-, una considerable expansión de los postgrados, en especial de las maestrías. Eso significó la incorporación de un nuevo ciclo a los ya heterogéneos modelos -el de las maestrías provenientes sobre todo de Estados Unidos-, sin una modificación sustancial de los objetivos y de la duración del primer ciclo de carácter profesional. Tal circunstancia ha llevado a algunos países (entre ellos Argentina) a una

${ }^{1}$ En Argentina, en el año 2002, las 92 universidades oficiales y privadas existentes otorgaron 4.219 títulos (muchos de ellos similares) entre los de grado y los de pregrado. La educación superior terciaria -o no universitaria- of reció otros 6.965 títulos, por lo que el total de los ofrecidos por la educación superior fue de 11.184. Fuente: N orberto Fernández Lamarra, La educación superior argentina en debate, Eudeba/IESALCUNESCO, Buenos Aires, mayo de 2003.

${ }^{2}$ En este artículo se utilizarán los términos vigentes en Argentina: Ias carreras de primer título (undergraduate) se denominan de grado (en otros países de América Latina de pregrado), y los posteriores al primer título, de postgrado (postgraduate). 


\section{N. FERNÁNDEZ}

duración total de alrededor de ocho a diez años formales -mucho más reales- de los dos ciclos: el de las licenciaturas o equivalentes, y el de las maestrías; es decir, se registró una superposición de dos modelos: el napoleónico, profesionalista, de carreras de grado largas, con el angloamericano, de maestrías posteriores a un ciclo corto, de cuatro años, como el Bachellor.

Si bien no hay información actualizada sobre el crecimiento de la matrícula de los postgrados ${ }^{3}$, se puede afirmar que han tenido un importante desarrollo cuantitativo en relación con el número de postgrados y de estudiantes. Así, en Argentina, el número total de carreras de postgrado pasó de 792 en 1994, a 1.054 en 1996, a 1.642 en 1999, y a 2.151 en 2003 , es decir, que casi se triplicaron en nueve años (Fernández Lamarra, 2003).

Este significativo crecimiento de los postgrados -en especial de las maestrías-, sin modificar el grado, genera una confusión entre los objetivos formativos de las carreras de grado y de postgrado, ya que la mayoría de éstos tiene una orientación profesionalista más o menos similar a la mayor parte de las ofertas académicas de grado.

A su vez, el crecimiento de los postgrados en muy pocos años ha significado una aún mayor heterogeneidad de los niveles de calidad, como ha ocurrido con el grado, y ha llevado a incluir su evaluación y su acreditación en los procesos de aseguramiento de la calidad puestos en marcha últimamente en casi todos los países de América Latina, tal como se verá en el próximo punto.

\section{EL ASEGURAMIENTO DE LA CALIDAD: EVALUACIÓN Y ACREDITACIÓN}

\subsection{LA DÉCADA DE LA CALIDAD EN AMÉRICA LATINA}

A finales de la década de los 80 y principios de la de los 90 , el tema de la calidad comenzó a afirmarse en el escenario y en la agenda de la educación en diversos países de América Latina. En décadas anteriores, los enfoques predominantes en materia de planeamiento y de

\footnotetext{
${ }^{3}$ EI IESALC de la UNESCO está llevando a cabo una serie de estudios nacionales sobre el postgrado que conducirán a un futuro estudio regional sobre el tema, lo que posibilitará disponer de mayor información y documentación sobre dichos procesos.
} 
desarrollo de la educación pusieron énfasis en los aspectos cuantitativos y en la vinculación con lo económico y con lo social. Los mayores desafíos para los sistemas educativos fueron su crecimiento, la ampliación de su cobertura, la construcción de escuelas, la formación de docentes, y la escasa atención a los aspectos de la calidad. Las reformas educativas de Ios 70 en varios Estados (Chile, Colombia, Costa Rica, Panamá, Perú, Venezuela, etc.) incluyeron la temática de la calidad, pero en el marco de las transformaciones de los sistemas educativos, que, en general, no tuvieron éxito ni continuidad.

En los años 80, y dentro del marco de las restricciones políticas impuestas en sus etapas finales por las dictaduras militares o en el de las recién recuperadas democracias, según los casos, se produjo en América Latina un proceso de reflexión, en el que el tema de la calidad, junto con el de la equidad, se asumieron como prioritarios. Ante todo, en lo concerniente a los nivel es primario y medio, y relacionado en especial con los operativos de medición de los rendimientos de los estudiantes. Después, para el ámbito universitario, a partir de los procesos de evaluación institucional llevados a cabo desde hace largo tiempo en Estados Unidos y Canadá, y más recientemente en Europa en el espacio de la constitución de la Unión Europea.

Todo esto dentro de una nueva concepción del Estado -que reemplazó a la del Estado del Bienestar-, en la que la rendición de cuentas (la accountability) se constituyó en un componente principal, en particular en la relación entre Estado y sociedad, y entre Estado, sociedad y universidad (el Estado Evaluador).

\subsection{CONCEPCIONES SOBRE LA CALIDAD DE LA EDUCACIÓN}

El papel primordial que desempeña la temática de la calidad y su evaluación lleva también a tener que discutir acerca de las distintas concepciones que sobre esto se han llevado a cabo en América Latina, en Estados Unidos y en Europa. Es conveniente que esa discusión se plantee tanto a nivel nacional como regional, tal como ocurre en Europa, y como sin duda lo va a ser en América Latina en el marco de los procesos de integración regional y de convergencia de sus sistemas educativos.

En Europa, la European Network for Quality Assurance (ENQA) y la J oint Quality Initiative han contribuido a dicho debate y a homogeneizar concepciones, estándares e indicadores de calidad en el ámbito 
de la Unión Europea. En Estados Unidos y Canadá -con una más larga tradición sobre esta temática- las concepciones y sus metodologías se revisan de forma periódica en función de los requerimientos sociales y profesionales.

En América Latina este debate está todavía pendiente, aunque se han hecho algunos progresos parciales. Incluso se encuentra sin resolver en cada uno de los países en los que, muchas veces, se asumen concepciones diferentes en distintos procesos de evaluación y de acreditación. Hasta el momento ha sido difícil y complejo alcanzar acuerdos sobre calidad de la educación, sobre eficiencia y sobre productividad.

Las concepciones sobre calidad varían según los actores. Para los académicos, se refiere a los saberes; para los empleadores, a competencias; para los estudiantes, a empleabilidad; para la sociedad, a ciudadanos respetables y competentes; para el Estado, según la concepción que asuma, puede variar: de aspectos vinculados con el desarrollo social y humano, a la eficiencia, a los costos y a los requerimientos de capital humano. En realidad -como señala Dias Sobrinho-, el concepto de calidad es una construcción social que varía según los intereses de los grupos de dentro y de fuera de la institución educativa, que refleja las características de la sociedad que se desea para hoy y que se proyecta para el futuro. No es un concepto unívoco y fijo, sino que debe ser construido a través de consensos y de negociaciones entre los actores (Dias Sobrinho, 1995). Es, por lo tanto, una concepción de construcción colectiva y gradual, que integra y articula visiones y demandas diferentes con los valores y con los propósitos de la institución educativa.

\subsection{LA EVALUACIÓN DE LA CALIDAD}

Así como ha existido y existe un debate sobre concepciones diferentes acerca de la calidad de la educación, también se encuentra una diversidad de enfoques sobre su evaluación. Para algunos, el énfasis en la concepción de la evaluación se produce en lo valorativo, en la emisión de juicios de valor; para otros, lo importante es la toma de decisiones; pero hay también ciertos autores que ponen el acento en la ética, en una evaluación al servicio de los valores públicos y de los justos intereses de los actores.

Para J acques L '́́cuyer, de Canadá, se debe evaluar para mejorar la calidad, descubriendo fortalezas y debilidades y tomando las 
decisiones necesarias; también se debe emitir un juicio de valor sobre la institución y sobre sus programas, fundamentado en bases sólidas, con criterios y estándares conocidos y aceptados, y teniendo en cuenta la misión y los objetivos institucionales.

Para Dilvo Ristoff, de Brasil, evaluar es una forma de restablecer compromisos con la sociedad; de repensar objetivos, modos de actuación y resultados; de estudiar, proponer e implementar cambios en las instituciones y en sus programas; se debe evaluar para poder planificar, para evolucionar.

Según la Comisión Nacional de Evaluación y Acreditación Universitaria (CONEAU), de Argentina, la evaluación debe servir para interpretar, para cambiar y para mejorar las instituciones y los programas, y realizarse en forma permanente y participativa; debe ser un proceso abierto, flexible y establecido en el marco de la misión y de los objetivos de la institución, y permitir conocer, comprender y explicar cómo funcionan las universidades para poder interpretarlas y mejorarlas y para producir innovaciones y cambios; debe contribuir al progreso de las prácticas institucionales, enriquecer la toma de decisiones, impulsar la comprensión que los actores tienen de la institución, y estimular la reflexión sobre el sentido y el significado de la tarea que realiza ( CONEAU).

\subsection{LA EVALUACIÓN DE LA CALIDAD EN AMÉRICA DEL NORTE Y EN EUROPA}

En América del Norte (Estados U nidos y Canadá) la evaluación ha constituido desde hace décadas una actividad permanente, en estrecha relación con la gestión de las instituciones. En Estados Unidos, la evaluación institucional en el nivel superior se lleva a cabo a través de agencias regionales, y la acreditación de carreras la hacen agencias especializadas. En Canadá, la evaluación-mejor dicho, la metaevaluaciónestá a cargo de los consejos provinciales de las universidades, y la acreditación de carreras se hace con criterios similares a los de Estados Unidos. México -como miembro del NAFTA- está incorporando criterios institucionales similares a los de Estados Unidos y Canadá.

En Europa, la constitución de la U nión Europea puso en marcha procesos de evaluación con fines académicos y profesionales en el marco de una tendencia creciente a la accountability y al control de calidad de productos, de servicios y de procesos (Total Quality Management) también en la educación superior. A mediados de la década de los 80 eso 
formó parte de mayores exigencias sociales de calidad en el ámbito de la sociedad del conocimiento y de la tecnología, de más autonomía para las universidades, de mayores requerimientos presupuestarios, $y$, por ende, de la puesta en marcha en Francia y en otros países de "contratosprograma» entre gobiernos y universidades.

Dichos procesos se han acelerado en los últimos años con la Declaración de Bolonia de 1999 y con la puesta en funcionamiento de agencias acreditadoras en varios países. La última Conferencia de Ministros de Educación, celebrada en Berlín en septiembre de 2003, consideró la evaluación y la acreditación como instrumentos clave para la construcción del Espacio Europeo de Educación Superior. Para ello se constituyó en Córdoba (España), en noviembre de 2003, el Consorcio Europeo de Acreditación.

Los enfoques de evaluación de la calidad utilizados en Europa han sido, en términos generales, los siguientes: el de Gran Bretaña, con estándares cuantitativos y cualitativos muy detallados y vinculando la evaluación con la asignación presupuestaria; el de Francia, de carácter institucional y asumiendo a la universidad en su conjunto; y el desarroIlado por Dinamarca, España, Holanda y otros países, con énfasis en la evaluación de carreras (titulaciones).

\subsection{LA EVALUACIÓN DE LA CALIDAD EN AMÉRICA LATINA}

Como ha sido señalado, la preocupación por la calidad y por su evaluación se inició en la región a fines de la década de los 80 y principios de la de los 90 . En la educación básica se presentó vinculada con los operativos nacionales y regionales de medición de los aprendizajes y de evaluación de la calidad, impulsados en la mayor parte de los países por la UNESCO y su Proyecto Principal de Educación. A nivel regional, la UNESCO impulsó la creación, en 1994, del Laboratorio Latinoamericano de Evaluación de la Calidad de la Educación, conformado por trece países, y que constituye una red para la realización de estudios comparativos, de investigaciones, de generación de estándares y de asistencia técnica horizontal.

En los últimos años algunos países latinoamericanos han participado en estudios internacionales ajenos a la región, como los de laOCDE, con resultados poco al entadores en relación con los de otras regiones del mundo. 
En la educación superior la preocupación por la evaluación se relaciona sobre todo con la situación ya señalada, en cuanto al gran crecimiento del número de instituciones y de matriculación y a la diversificación institucional con niveles muy heterogéneos de calidad. Por descontado que en eso también ha influido el desarrollo de estas actividades en Europa, y los procesos de integración regional como el NAFTA y el MERCOSUR.

En México se creó, en 1989, la Comisión Nacional para la Evaluación de la Educación Superior (CONAEVA), en el seno de la Coordinación Nacional para la Planeación de la Educación Superior (CONPES); en Chile se fundó, en 1990, el Consejo Superior de Educación, destinado a las instituciones privadas no autónomas (nuevas universidades privadas); en Colombia se instituyó, en 1992, el Consejo Nacional de Acreditación (CNA); en Brasil se puso en marcha, en 1993, el Programa de Evaluación Institucional de las Universidades Brasileñas ( PAIUB), por iniciativa de las propias universidades en acuerdo con el Ministerio de Educación; en Argentina se estableció la Comisión Nacional de Evaluación y Acreditación Universitaria (CONEAU) en 1995/96. A finales de la década de los 90 y principios de la actual, surgieron nuevos organismos y proyectos de evaluación y de acreditación universitaria en otros países (por ejemplo, en Bolivia, Costa Rica, Ecuador, El Salvador, Nicaragua y Uruguay), yen subregiones como Centroamérica (el Sistema Centroamericano de Evaluación y Acreditación de la Educación Superior, SICEVAES, en 1998) y el MERCOSUR (el Mecanismo Experimental de Acreditación de Carreras para el Reconocimiento de Grado Universitario, aprobado en 1998). En enero de 2003 se creó la Agencia Nacional de Evaluación y Acreditación de la Educación Superior en Paraguay. En fecha muy reciente, abril de 2004, el Congreso de Brasil aprobó una Ley Federal creando el Sistema Nacional de Evaluación de la Educación Superior (SINAES).

En México se organizó, en el año 2000 , el Consejo para la Acreditación de la Educación Superior (COPAES), con fines específicos de acreditación de programas académicos de nivel superior (carreras 0 titulaciones), a partir de la experiencia en la década de los 90 de la CONAEVA, de los Comités Interinstitucionales para la Evaluación de la Educación Superior (CIEES) y de diversos consejos, comisiones o asociaciones profesionales con funciones de acreditación y de certificación al estilo de las existentes en Estados Unidos, ya que estos procesos estuvieron enmarcados en la integración de México al NAFTA. En Chile fue creada, en marzo de 1999, la Comisión Nacional de Acreditación de 
Pre-grado (CNAP). En Argentina, la CONEAU puso en marcha procesos de acreditación de carreras de grado y de postgrado y de instituciones.

Esta breve caracterización de la evolución en la década de los 90, y de la situación actual en materia de evaluación y de acreditación universitaria en los países de América Latina, permite extraer algunas conclusiones preliminares:

- Se ha avanzado en relación con la «cultura de la evaluación» en la educación superior en la mayoría de los países, superando en gran medida las tensiones planteadas en los primeros años de la década de los 90 en cuanto a autonomía universitaria versus evaluación.

- El mayor desarrollo se ha registrado en lo referente a la evaluación diagnóstica para el mejoramiento de la calidad y de la pertinencia institucional y no en lo que tiene que ver con fines de acreditación.

- Los procesos tendientes a la acreditación de carreras de grado, a partir de criterios y de estándares preestablecidos, comienzan a ser desarrollados en los últimos años de la década de los 90 como un paso más al de los procesos de evaluación para el mejoramiento de la calidad, y en su mayoría se encuentran actualmente en etapa experimental.

- Los procesos de acreditación de los postgrados cuentan con una amplia trayectoria en Brasil, y han sido aplicados de forma masiva en Argentina; para programas de carácter regional, también en Centroamérica a través del SICAR (Sistema de Carreras y Postgrados Regionales); en México y en otros países, vinculados con la asignación de recursos financieros del sistema científico-tecnológico.

- Los procesos tendientes a la acreditación institucional, es decir, para la aprobación de nuevos centros universitarios privados o para la revisión de su funcionamiento, han posibilitado en varios países - como Argentina, Chile, Colombia y Uruguay- limitar la proliferación excesiva de nuevas instituciones universitarias, y tender a una mayor homogeneidad en cuanto a los niveles de calidad. Así, en Argentina, 
en la primera mitad de la década de los 90 , se aprobaron 23 nuevas universidades privadas, mientras que desde 1995 hasta este año sólo 10, gracias al funcionamiento de la CONEAU. En Chile se crearon, hasta la instalación del Consejo Superior de Educación, 43 nuevas universidades privadas, y desde ese momento (entre 1990 y 2000) sólo cinco; la labor de este Consejo ha llevado también a disponer el cierre de siete instituciones.

- Como se verá más adelante, han sido muy significativos los avances producidos en los últimos años en materia de acreditación de carreras y de títulos para el reconocimiento regional: en el MERCOSUR, con la puesta en marcha del Mecanismo Experimental deAcreditación de Carreras( MEXA); en Centroamérica, con la creación del Consejo Centroamericano de Acreditación y de varias redes de facultades en las áreas de ingeniería, medicina y agronomía; en el NAFTA, con la implementación en México de procesos de acreditación de carreras con procedimientos y con criterios similares a los vigentes en Estados Unidos y Canadá.

\section{LOS PROCESOS DE CONVERGENCIA EN AMÉRICA LATINA}

\subsection{LA NECESIDAD DE LA CONVERGENCIA}

Como ya ha sido señalado, hay falta de convergencia en las políticas de educación superior en América Latina por la influencia de modelos muy distintos, muy puntuales, muy heterogéneos. Se registra una importante divergencia en cuanto a lo institucional: existen desde emblemáticas y tradicionales universidades hasta muy pequeños centros, que, en algunos países, se conocen como «universidades garajes». También se dan situaciones diversas en materia de calidad de la educación superior y de su evaluación. J ustamente ahora me encuentro elaborando el estudio regional para el IESALC de la UNESCO sobre los procesos de aseguramiento de la calidad de la educación superior en América Latina y el Caribe, tanto de evaluación como de acreditación. Uno de los puntos esenciales en este análisis es la falta de coherencia sobre tales temas: influencias múltiples en materia de diseños, de organización de los postgrados, de educación a distancia y virtual. 
También en dicho marco aparece la educación transnacional, sin regulaciones ni controles adecuados, y, así mismo, con model os muydisímiles.

En cuanto al diseño de los modelos académicos, se han producido cambios sustantivos: hasta las décadas de los años 70 y 80 existía un predominio de carreras profesionales de model o napoleónico. A partir de esa etapa se registró una ampliación y una diversificación de la oferta con criterios académicos distintos y a veces contradictorios: por ejemplo, la supervivencia de las carreras tradicionales largas - «carreras túneles» en términos del lenguaje europeo- con seis años formales y ocho reales, frente a carreras más cortas y de dudosa «profesionalidad». En otras áreas se definieron los títulos de manera poco tradicional. En Argentina, v. gr., en el área de Ingeniería, hay más de 100 títulos diferentes. Se observa así la supervivencia de distintos modelos. En general, las carreras se conforman por adiciones, por agregados o por parches que provienen de tan diversos orígenes y que muestran influencias disímiles y contrapuestas, lo que causa dificultades para la compatibilidad, para la convergencia, y, también, para la movilidad de los estudiantes ante un panorama tan fragmentado. De ahí la necesidad de debatir sobre objetivos académicos, sobre alcance de los títulos y sobre articulación institucional.

Como ya se dijo, en tiempos más recientes se han desarrollado las carreras de postgrado con fuerte influencia del modelo master norteamericano, pero sin que se haya modificado sustancialmente el grado. Por lo tanto, se produce cierta incompatibilidad y cierta confusión de roles entre el grado y el postgrado, y una presencia cada vez más significativa de ofertas extranjeras como producto de la educación transnacional, sin regulaciones adecuadas en varios países. Por ejemplo, en Argentina, las carreras de grado son largas y también lo son los postgrados. Se ha estimado que los ocho o nueve años formales para el grado y la maestría, se transforman en diez, en doce o en más reales. De ahí la necesidad de replantear la articulación del grado y del postgrado en cuanto al alcance de los títulos, a los niveles de formación, a la habilitación profesional, y a la diferenciación o no entre carreras académicas y profesionales. En Argentina y en al gunos otros países de América Latina - por fortuna no se trata de una situación general en la región- hay un escaso crecimiento en el nivel de doctorado, lo que afecta el desarrollo académico deseable. 


\subsection{ALGUNOS PROCESOS DE INTEGRACIÓN REGIONAL EN MARCHA}

Los procesos de integración regional que se vienen registrando en el área en las últimas décadas han planteado la necesidad de atender a la articulación y a la convergencia de los sistemas, y, particularmente, al reconocimiento de títulos y de estudios a fin de posibilitar la movilidad de las personas en el marco de cada región.

Uno de estos procesos ha sido el de la integración delMERCOSUR, con Argentina, Brasil, Paraguay y Uruguay como fundadores, y la posterior incorporación de Bolivia y de Chile como asociados. Recientemente han solicitado su incorporación México, Perú y Venezuela. Otro de los procesos es el del NAFTA, con la integración de México con Estados Unidos y Canadá. En Centroamérica se han registrado avances, a pesar de los problemas políticos que dificultaron en décadas anteriores la consolidación de la región. Los países andinos también tuvieron complicaciones para consolidar el Pacto Andino, que funcionó más o menos bien hasta finales de la década de los 70 y comienzos de la de los 80; en estos países hay que destacar la organización y el funcionamiento del Convenio Andrés Bello para atender los aspectos de articulación en materia de educación y de cultura.

Los procesos de integración regional son indicadores muy positivos dirigidos hacia una meta deseable y necesaria, que fue un muy loable ideal del Libertador Simón Bolívar al comienzo de los procesos de independencia de los países latinoamericanos: lograr su unidad. Transcurridos casi dos siglos, es de esperar que los procesos de integración regional coadyuven a estos propósitos, ahora casi imprescindibles frente a la globalización y a la internacionalización de los aspectos políticos, sociales, económico-productivos, culturales y educativos a nivel mundial.

En el punto 4 de este artículo se desarrollará lo referente a los procesos de convergencia y de articulación en educación en el ámbito del MERCOSUR, sobre todo en cuanto a acreditación de carreras universitarias. Los avances en dicha área pueden ser de utilidad para otras de la región. 


\subsection{OTROS PROGRAMAS Y PROYECTOS DE CONVERGENCIA}

En los últimos años diversos organismos de cooperación internacional, regional o bilateral, han iniciado programas y proyectos que han promovido la convergencia y la articulación entre los sistemas de educación superior. Entre ellos se pueden citar laUNESCO (en particular a través del IESALC); Ia OEI; la Organización Universitaria Interamericana (OUI), sobre todo por medio del Instituto de Gestión y Liderazgo Universitario (IGLU); Ia Comisión Europea (mediante los proyectos ALFA y ALßAN); IOS organismos de cooperación de países como Canadá, España, Estados Unidos, Francia...; el programa Columbus, etcétera.

También se organizaron redes interuniversitarias o interagenciales que han coadyuvado a estos propósitos de convergencia: el Grupo Montevideo, el CSUCA, el CINDA, la Red Latinoamericana de Cooperación Universitaria, la Asociación de Universidades de América Latina y el Caribe para la Integración (AUALCPI), la Red Iberoamericana para la Acreditación de la Calidad de la Educación Superior (RIACES), etcétera.

En el punto 5 se caracterizarán estos programas y proyectos de convergencia y articulación.

\section{CONVERGENCIA Y ARTICULACIÓN EN EL ÁMBITO DEL MERCOSUR}

\subsection{EL MECANISMO EXPERIMENTAL DE ACREDITACIÓN DE CARRERAS (MEXA). ANTECEDENTES}

EI MERCOSUR incluyó desde sus comienzos el Área Educación como uno de sus aspectos prioritarios, e instituyó la Reunión de Ministros de Educación (RME) como ámbito de decisiones sobre dicha temática. Desde su primer Plan Trienal de Educación se planteó el reconocimiento y la acreditación de estudios y de títulos entre sus objetivos principales, para posibilitar la movilidad de las personas dentro de la región. Esta característica de «precocidad» en cuanto a la consideración de los aspectos educativos en el proceso de integración regional ha sido reconocida por múltiples especialistas, incluso europeos (Fernández Lamarra y Pulfer, 1992; Lémez, 2002).

En tal sentido, avanzó más rápidamente en relación con el reconocimiento de títulos y de estudios para la educación primaria o 
básica y para la enseñanza media general y técnica, ya que estos procesos de reconocimiento eran más sencillos. Como señala Lémez, se adoptó un criterio de construcción de carácter gradual, de abajo hacia arriba, de planteamiento y solución de problemáticas de complejidad creciente. Al mismo tiempo, con la aprobación de los protocolos de títulos y de estudios para los niveles primario y medio, se establecieron paradigmas compatibles en temas significativos para el proceso de integración, como son los referidos a la enseñanza de la historia y de la geografía 4 .

Las dificultades para el reconocimiento de los títulos y de los estudios de nivel superior derivan de las estrechas y complejas vinculaciones existentes entre la validación de los títulos profesionales y el ejercicio de las respectivas profesiones, ya que -como señala Lémezresponden a lógicas diferentes, muchas veces opuestas al espíritu aperturista del sector educación, y que involucran a actores nacionales de muy diversa índole, con representaciones y con intereses muy disímiles. En un estudio llevado a cabo para la Organización Internacional para las Migraciones (OIM) se han analizado los mecanismos y las diferentes situaciones existentes en Argentina, Brasil, Paraguay y Uruguay con respecto a las habilitaciones para el ejercicio profesional, con el funcionamiento de consejos profesionales de muy diversa estructuración en Argentina y Brasil, y con la no existencia de los mismos en Paraguay y U ruguay (Fernández Lamarra, 1999). Por otra parte, lo referido a estos temas de servicios personales en la región depende de un grupo de trabajo del Consejo del Mercado Común, y la RME no tiene incumbencias en ellos.

La discusión enrareció los debates yen un principio dificultó su análisis, hasta que, poco a poco, partiendo de acuerdos tácitos y luego en forma explícita, se decidió abocarse a su resolución desde distintos ángulos y perspectivas y desde diferentes niveles de especificidad (Lémez, 2002).

Se asumió que el reconocimiento de títulos y de estudios universitarios tenía dos objetivos principales: uno era de carácter académico, para posibilitar la continuación de estudios, para cursar el postgrado

${ }^{4}$ En una investigación dirigida por el autor en el ámbito de la Universidad Nacional de La Plata, se estudiaron los programas de historia y de geografía y los libros de texto para los niveles primario y medio de los países del MERCOSUR, estableciéndose los importantes vacíos temáticos y las fuertes discrepancias en las explicaciones y en las interpretaciones de los mismos hechos históricos y geográficos en cada uno de los sistemas educativos. 
y para desempeñar actividades académicas; el otro consistía en habilitar para el ejercicio profesional en país diferente al que había otorgado el título. El primero era más simple y se pudo desarrollar en forma concreta; el segundo más complejo, porque se relaciona con el rol del Estado y con su obligación de asegurar la formación recibida con el fin de habilitar para el desempeño profesional, aún más cuando se trata de profesiones que se vinculan con la salud, con los bienes de los habitantes y con la seguridad. En relación con este segundo objetivo, se debe articular con las regulaciones vigentes para el desempeño profesional específico, con situaciones disímiles según los casos, tal como ya fue expuesto.

El reconocimiento para el desempeño de actividades académicas tuvo un rápido avance, ya que se firmaron dos protocolos: el de Integración educativa para la prosecución de estudios de postgrado en las universidades de los países $m$ iembros del MERCOSUR, aprobado en Montevideo en noviembre de 1995, y el de Admisión de títulos y grados universitarios para el ejercicio de actividades académicas en los países del MERCOSUR, firmado en Asunción en junio de 1997. También en noviembre de 1995 se aprobó el Protocolo de Integración educativa para la formación de recursos humanos a nivel de postgrado, tendiendo a una formación comparable y equival ente y a la adaptación de los ya existentes en los países del MERCOSUR. En este Protocolo se establecen los criterios para definir los títulos de postgrado (especialización, maestría y doctorado) para la acreditación de los respectivos programas, tanto de orientación profesional como académica, y los referidos a la acreditación de postgrados interinstitucionales.

La situación más compleja y difícil se presentó cuando se trató el tema del reconocimiento destinado a habilitar para el desempeño profesional. En el ámbito de la Comisión Técnica Regional de Educación Superior (CTR-ES) se planteó, en primer lugar, un proceso basado en tablas de equivalencias, ya ensayado en convenios anteriores. Se acordó el establecimiento de grupos de trabajo por cada carrera, de las que se seleccionaron inicialmente Abogacía y Contaduría Pública, y, más tarde, Ingeniería. Tras las primeras reuniones de trabajo, pudo comprobarse que este régimen era de muy difícil instrumentación.

En noviembre de 1996, en la XI Reunión de Ministros realizada en Gramado, Brasil, se resolvió dejar de lado el proceso basado en tablas de equivalencias, y considerar la posibilidad de instrumentar otro fundado en la acreditación de carreras de grado. Para ello se creó, en junio de 1997, el Grupo de Trabajo de Especialistas en Evaluación y Acreditación 
de la Educación Superior, encomendándosele la elaboración de una propuesta de organización de un sistema de acreditación de instituciones y de carreras que permitiese facilitar el reconocimiento de títulos de grado.

En junio de 1998 los Ministros de Educación de los países del MERCOSUR, más los de Bolivia y Chile, aprobaron un Memorando de Entendimiento para la Implementación de un Mecanismo Experimental para la Acreditación de Carreras de Grado. Ese Memorando plantea los principios generales, los criterios para la determinación experimental de las carreras, y los procedimientos para la acreditación, sus alcances e implicaciones.

\subsection{EL MECANISMO DE ACREDITACIÓN MERCOSUR (MEXA). NORMAS GENERALES}

El Memorando de Entendimiento aprobado por la RME, planteó, entre otros, los siguientes puntos:

- Otorgar validez a los títulos de aquellas carreras que lo requieran para el ejercicio de la profesión.

- Adoptar un sistema de libre adhesión, periódico, de aplicación gradual y experimental.

- Incluir un paso previo de autoevaluación.

- Establecer un proceso de evaluación por pares, de acuerdo con los requisitos establecidos a nivel regional.

- Los requisitos se referirán a la carrera en su integridad (planes de estudio, cuerpo docente, infraestructura y equipamiento, etc.).

- Los organismos competentes a nivel nacional serán las Agencias Nacionales de Acreditación, que deberán cumplir los siguientes requisitos:

- Ser personas de derecho público debidamente reconocidas por las autoridades nacionales.

- Tener carácter pluripersonal. 
- El procedimiento para su integración deberá garantizar la idoneidad de sus miembros y la autonomía de sus decisiones.

- Se establece la Reunión de Agencias de Acreditación del MERCOSUR como instancia de monitoreo y de cooperación regional.

En octubre de 1998 se reunió en Brasilia el Grupo de Trabajo de Especialistas en Evaluación y Acreditación ( GTEEA) para elaborar una propuesta destinada a la implementación del Memorando de Entendimiento. En dicha reunión se analizaron los mecanismos de acreditación utilizados por cada país con el fin de establecer si respondían o no a los criterios y a las características planteadas en el Memorando, y para saber cuál era la situación de los países que no tenían organismos o procedimientos creados con tal propósito.

A partir de este análisis -y a pesar de situaciones aún no definidas a nivel nacional- se establecieron criterios para la constitución de Comisiones Consultivas Regionales de especialistas por carrera, a través de un documento de Directrices Generales de Operación de dichas comisiones. En el documento se establecieron, entre otros aspectos, los referentes a los criterios de selección de los expertos que participarían por cada país en la elaboración de criterios de evaluación y de estándares para la acreditación de las carreras seleccionadas; al respecto, tendrían que ser docentes universitarios, con título de postgrado o reconocimiento equivalente, y con experiencia profesional y académica. También se determinó acerca de la composición de las comisiones consultivas y sobre la elaboración de los términos de referencia. Con respecto a las categorías de evaluación, se sugirió incluir por lo menos las siguientes: proyecto del curso, estructura curricular, administración académica, cuerpo docente, biblioteca, infraestructura, equipamiento, materiales, componentes de autoevaluación, y cooperación interinstitucional.

En cuanto a los Comités de Pares, se acordó que deberían tener en cuenta los criterios y los parámetros de calidad que se hubieran fijado para cada carrera. En su integración se determinó, inicialmente, que debería participar al menos un especialista de cada uno de los países del MERCOSUR. Más tarde, en junio de 2001, se redujo a dos pares de otros países en cada comité, que tendrían que ser convocados por la respectiva Agencia Nacional. 
Así mismo, se estableció que el mecanismo se aplicaría de forma experimental y gradual en carreras en las que el título universitario fuera requisito o condición para el ejercicio de la profesión, aunque con la aclaración de que el reconocimiento académico del título «no conferirá de por sí el derecho al ejercicio de la profesión», para distinguir el proceso de acreditación de las regulaciones que se adopten en materia de desempeño profesional. Se determinó que el lapso máximo para la primera acreditación sería de cinco años, y que se extendería hasta que se llevara a cabo el segundo proceso si la solicitud para el nuevo plazo fuera presentada en tiempo y en forma.

La RME realizada en Brasilia en noviembre de 1998, resolvió que el Mecanismo Experimental se iniciara para las carreras de Medicina, de Ingeniería y de Agricultura, según lo propuesto por las Comisiones Técnicas Regionales de Educación Superior y de Postgrado, y por el Grupo de Trabajo de Especialistas en Evaluación y Acreditación ( GTEEA).

\subsection{REGLAMENTACIONES SOBRE EL PROCESO DE ACREDITACIÓN MERCOSUR}

El Memorando de Entendimiento se complementa con un Anexo, que contiene las Normas Generales de Operación y los Procedimientos del MEXA. Este Anexo, compuesto de ocho capítulos, detalla, entre otros aspectos, los siguientes:

- Solicitud de acreditación, en la que la institución solicitante debe declarar explícitamente que cumple con los requisitos y que acepta los criterios y las normas establecidos, y presentar el informe de autoevaluación accediendo a la visita del Comité de Pares y al dictamen que corresponda.

- La estructura general del informe de autoevaluación. Según sus normas, este informe es el resultado de «un proceso mediante el cual la carrera o programa que busca la acreditación da cuenta del grado en que se ajusta a los criterios y parámetros de calidad establecidos y concordados para ella, así como de la forma en que cumple con sus propias metas y propósitos».

Según estas pautas, el informe debe contener, entre otra información y documentación, lo referido al desempeño de la carrera en 
relación con los estándares establecidos, la descripción de fortalezas y debilidades, el contexto institucional, el proyecto académico, la autoevaluación acerca del cumplimiento de los criterios y parámetros de calidad fijados para la carrera por el MERCOSUR, y el respaldo documental con la información cuantitativa y cualitativa necesaria, los reglamentos y los convenios pertinentes, etcétera.

\subsection{CONSTITUCIÓN DE LOS COMITÉS DE PARES}

Cada Agencia Nacional de Acreditación (ANA) designará al Comité de Pares, que entenderá en cada carrera que solicite acreditación al MERCOSUR; deberá estar integrado, como mínimo, por tres representantes, e incluir no menos de dos delegados de países distintos al que pertenece la carrera, que serán convocados por la Agencia de su país a partir del registro de evaluadores.

Las principales tareas de los Comités de Pares son las siguientes:

- Validar el informe de autoevaluación.

- Evaluar el grado en el que la carrera se ajusta a los criterios y a los parámetros establecidos.

- Evaluar el grado de cumplimiento de las metas y de los objetivos que se han definido.

\subsection{ELABORACIÓN DE LOS CRITERIOS Y DE LOS INDICADORES PARA LAACREDITACIÓN DE LAS CARRERAS}

De acuerdo con lo expuesto más arriba, se dispuso que el MEXA se iniciara con las carreras de Medicina, de Ingeniería y de Agronomía. Para ello se constituyeron las respectivas comisiones consultivas de expertos, con representantes de las universidades y de las asociaciones profesionales de cada uno de los países.

Como criterio general, se adopto el de organizar los documentos de cada una de las tres carreras en componentes, y para cada uno de ellos se establecieron los criterios, los indicadores y las respectivas fuentes de información. Los componentes adoptados fueron: contexto institucional, proyecto académico, población universitaria, e infraestructura. 


\subsection{PUESTA EN MARCHA}

La RME dispuso en el año 2002 la puesta en marcha del Mecanismo Experimental en las carreras de Agronomía, de Ingeniería y de Medicina, en ese orden. Ya se llevaron a cabo las respectivas convocatorias, y se evaluarán cinco carreras de cada especialidad en cada país, que se hayan presentado voluntariamente. En los casos en los que el número de solicitudes sea superior a cinco, se seleccionarán por la antigüedad institucional de cada una de ellas.

EI MEXA está concluyendo las acreditaciones para Agronomía, y se están iniciando para Medicina y para Ingeniería. Se responsabilizan como ANAs de esta tarea la CONEAU en Argentina, la CNAP en Chile, el Consejo Superior de Educación en Brasil, la recientemente creada Agencia Nacional de Evaluación y Acreditación en Paraguay, y los respectivos Ministerios de Educación en Bolivia y Uruguay.

\section{OTROS PROGRAMAS Y PROYECTOS}

\subsection{LOS ESTUDIOS DEL IESALC DE LA UNESCO}

El Instituto Internacional para la Educación Superior en América Latina y el Caribe (IESALC) de la UNESCO, con sede en Caracas, está desarrollando en los últimos años una serie de trabajos y de estudios nacionales y regionales sobre la educación superior, que contribuyen con vigor a la convergencia de los sistemas nacionales y a la articulación de sus políticas. Para ello, desde junio de 2001 ha puesto en marcha el Observatorio de la Educación Superior en América Latina y el Caribe, con el que pretende generar un ámbito de reflexión al más alto nivel sobre la problemática universitaria y su transformación, abrir un espacio de sensibilización y de intercambio de información digitalizada especializada, dar acceso regular al acervo de informaciones y de datos de dominio público sobre educación superior, y facilitar la socialización del conocimiento y la convergencia de los sistemas.

Por todo eso, el IESALC ha generado una importante colección de estudios nacionales sobre educación superior, tanto de sus aspectos generales como sobre facetas específicas. En los estudios nacionales se han concluido al rededor de diez, en su mayoría ya editados. Antes de su versión definitiva, el IESALC organiza talleres nacionales para que auto- 
ridades educativas y universitarias y expertos puedan brindar sus aportes al estudio elaborado por un especialista destacado del país. Estos estudios abarcan la evolución y el contexto histórico de la educación superior en cada país; su relación con el contexto nacional e internacional; la descripción detallada del sistema; el gobierno y la gestión; los actores de la educación superior (estudiantes, docentes, egresados, no docentes, etc.); las estructuras académicas vigentes de grado y de posgrado; la educación a distancia; los procesos de evaluación y de acreditación; la investigación científico-tecnológica; el financiamiento, y los principales avances en materia de innovaciones y de reformas. Tales trabajos constituyen una buena base para promover estrategias de convergencia y de articulación entre los países de América Latina.

Además de estos estudios, el IESALC ha promovido la realización de análisis por países sobre otros temas, que permiten una mayor profundización del conocimiento sobre la educación superior en la región. La mayor parte de ellos ya está disponible en Internet. Para cada tema el IESALC promueve la elaboración de un estudio comparativo regional, que se discute en un seminario regional específico. Las temáticas abarcadas hasta ahora han sido las siguientes: evaluación y acreditación de la calidad, reformas en la educación superior, legislación vigente y su evolución, internacionalización y nuevos proveedores y modalidades, universidades indígenas, universidades religiosas, macrouniversidades y universidades pedagógicas. También ha realizado exámenes que profundizan otros temas, como los de género en la educación superior.

\subsection{LOS TRABAIOS Y PROYECTOS DE LA ORGANIZACIÓN DE ESTADOS IBEROAMERICANOS (OEI)}

La OEI también desarrolla una tarea contributiva a la convergen-

ciaylaantiailaciónentrelossistem aseducativosyalaeducación superior delepacioiberom ericano. IaRevista Iberoamericana de Educación, las colecciones de libros -en especial Cuadernos de Iberoamérica y la serie «Educación Comparada» de Cuadernos de la OEI-, así como otras publicaciones y trabajos, facilitan el conocimiento mutuo entre los países y sus sistemas educativos, favoreciendo el desarrollo de políticas y de proyectos compartidos.

En el ámbito de la educación superior, la OEI está promoviendo estrategias de cooperación interuniversitaria entre los países de la región, 
que están contribuyendo de forma paulatina a su articulación. Entre los programas que desarrolla se puede mencionar el PIMA (Proyecto de Intercambio y Movilidad Académica), que consiste en el intercambio deestudiantes deuniversidades del áreaen un cada vezmayor núm ero de dom inios. Com o ha dem ostradb la experiencia uropen, el intercambio de estudiantes es una estrategia my eficiente para prom over los procesos de integración regional, la convergencia de los sistem as de educación superior, y la antiaulación entre las instituciones universitarias, con oes el caso del program a Sócrates frasmus.

EIPIMA -iniciado por laOEl en 1999- es un programa multilateral organizado en redes temáticas con participación de universidades españolas, portuguesas y de América Latina. Está dirigido a estudiantes de grado (pregrado para otros países), y se basa en los siguientes puntos: la significación de la movilidad de los estudiantes, el trabajo en red, la focalización en áreas temáticas, y el reconocimiento de los estudios.

\subsection{LOS PROGRAMAS Y ACCIONES DE LA ORGANIZACIÓN UNIVERSITARIA INTERAMERICANA (OUI)}

La OUI fue fundada en 1979 en Quebec, Canadá, donde se encuentra su sede. Es una organización internacional dedicada a la cooperación entre las instituciones universitarias, y al desarrollo y articulación de la educación superior en toda América, desde Canadá en el norte, hasta Argentina y Chile en el sur. Está integrada por más de 400 afiliados: universidades públicas y privadas, centros de investigación, asociaciones universitarias nacionales o regionales, consejos de rectores, etcétera.

Sus actividades se llevan a cabo a través del Instituto de Gestión y Liderazgo Universitario (IGLU) y del Colegio de las Américas (COLAM). Las acciones de ambos organismos han influido con fuerza en los procesos de convergencia y articulación de la educación superior en América Latina, en especial mediante los cursos que imparte y otras actividades de formación y de cooperación.

Desde su creación en 1983, el IGLU ha capacitado a al rededor de 1.300 dirigentes universitarios, valiéndose de sus cursos de carácter subregional. Entre los objetivos de estos cursos están -además de los de capacitación para el liderazgo y la habilitación en gestión universitarialos de favorecer el intercambio entre los participantes de diversos países 
e instituciones, los de promover los estudios comparados, los de difundir los resultados de estudios e investigaciones llevados a cabo en las tres Américas y en Europa, etcétera.

Con independencia de sus cursos, el IGLU organiza seminarios y talleres sobre temas específicos, ya sea por propia iniciativa o por las propuestas que pueda recibir de establecimientos miembros. En estas actividades se profundizan tanto los aspectos de carácter político y estratégico, como los vinculados con lo metodológico de la gestión institucional, académica y administrativa.

EI Colegio de las Américas (COLAM) fue creado en 1997, y sus actividades se refieren a aspectos relacionados con la cooperación universitaria sobre temas de interés hemisférico y de integración continental, favoreciendo la comprensión intercultural, el uso pedagógico de las nuevas TIC, la interdisciplinariedad y las conexiones interamericanas. Sus labores las desarrolla a través de los denominados Seminarios Interamérica, destinados a estudiantes de diversos países y de distintas disciplinas, y de las Redes Interamericanas de Formación (RIF). Actualmente funcionan al rededor de diez redes sobre temáticas muy diversas de interés continental.

Hasta el año 2003 se habían llevado a cabo 17 Seminarios Interamérica, con más de 700 estudiantes; 62 universidades habían integrado las RIF, con la participación en sus programas de formación de más de 1.200 estudiantes.

\subsection{LA RED IBEROAMERICANA PARA LA ACREDITACIÓN DE LA CALIDAD DE LA EDUCACIÓN SUPERIOR (RIACES)}

Esta Red se constituyó en mayo de 2003 por las agencias de evaluación y acreditación de la calidad de diversos países de América Latina, por la ANECA de España, y por ministerios de educación de la región, con el apoyo de organismos internacionales como la OEI. Sus objetivos se centran en contribuir a la convergencia y a la articulación de la educación superior en el espacio iberoamericano, ya que se propone el desarrollo de proyectos y de acciones de intercambio, de conocimiento mutuo y de trabajo conjunto.

La Red está organizada por comisiones de trabajo sobre temas específicos: estudios comparados; gestión y mantenimiento de un portal 
propio (http://www.riaces.org); diseño de unsoftware para autoevaluación; talleres de formación para evaluadores; glosario de términos de evaluación y de acreditación; buenas prácticas en evaluación y acreditación; y estudio comparativo de títulos.

\subsection{LOS PROGRAMAS ALFA Y ALßAN DE LA COMISIÓN EUROPEA}

Estos programas se han constituido en los últimos años como instrumentos muy significativos para la articulación y la convergencia en materia de educación entre los países de la Comisión Europea y los de América Latina.

Los proyectos ALFA (América Latina-Formación Académica) son propuestas de trabajo en red entre instituciones universitarias europeas y latinoamericanas. Dichas redes temáticas están constituidas, al menos, por seis universidades de países diferentes: tres europeas y tres de los 18 países de América Latina, coordinadas por una de ellas.

Desde el año 2000 se han llevado a cabo ocho rondas, en las que se han aprobado 197 proyectos. Por el momento, están programadas otras cuatro hasta octubre de 2005. El análisis de los proyectos aprobados -ya ejecutados o en vías de ejecución- muestra una interesante diversidad temática, de instituciones coordinadoras, de países y de universidades participantes. Estas redes han producido una valiosa documentación, a la que puede accederse a través de las respectivas páginas web y de trabajos editados. La nómina completa de proyectos y una síntesis de los mismos puede consultarse en http://europa.eu.int/ comm/europeaid/proyects/alfa/information/compendium_es.pdf.

Entre los proyectos llevados a cabo puede señalarse el de Acreditación y Reconocimientos Oficiales entre Universidades del MERCOSUR y la UE (ACRO), iniciado en febrero de 2002, que está finalizando en la actualidad. Sus objetivos han sido: desarrollar marcos conceptual es de acreditación en los contextos europeo y latinoamericano (sobre todo en el MERCOSUR), establecer criterios comunes para el reconocimiento de títulos entre las universidades participantes, y proponer el establecimiento de mecanismos estables y generalizables a otras universidades de ambas regiones para la acreditación y el reconocimiento de títulos. 
EI Proyecto ACRO fue coordinado por la Universidad española de Valencia a través de J osé-Ginés Mora, y estuvo integrado por universidades de Argentina, Brasil, España, Holanda, Italia, Portugal y U ruguay. $\mathrm{Ha}$ producido una importante documentación sobre los procesos de evaluación y de acreditación en la educación superior en Europa y en América Latina, y en cada uno de los países miembros ( http://www.uv.es/alfa-acro) Este Proyecto tuvo como actividad de cierre la realización del Congreso Internacional América Latina y Europa ante los procesos de convergencia de la Educación Superior, que se llevó a cabo en la Universidad Nacional de Tres de Febrero (coordinadora para América Latina del Proyecto ACRO) en Buenos Aires, en junio de 2004. En dicho Congreso participaron más de 400 especialistas de países latinoamericanos -en especial de Argentina y de otros países del MERCOSUR-y de Europa, y se presentaron alrededor de veinte importantes ponencias. Sus conclusiones constituyeron un aporte decisivo para la consolidación del Espacio Latinoamericano de Educación Superior y para su convergencia con el Europeo.

EI ALßAN es un programa de becas de al to nivel para estudiantes y para profesionales de América Latina que deseen realizar estudios de postgrado (maestrías y doctorados) o de formación superior especializada en universidades de los países de la Unión Europea. Se inició en el año académico 2003-2004, en el que se asignaron 251 becas. En el curso 2004-2005 se han otorgado 779 becas: 317 para maestrías, 430 para doctorados, y 32 para especializaciones. Las áreas de estudio que han recibido mayor cantidad de becas han sido Ingeniería y Tecnología con 132, y Ciencias Sociales con 117. Del total de beneficiarios fueron a España 324, al Reino Unido 149, ya Francia 107. La nómina completa de becas otorgadas se puede consultar en http://www. programalban.org/ statis/estadisticas.

\subsection{REDES DE UNIVERSIDADES}

En las últimas décadas se han constituido diversas redes de universidades, tanto entre las de América Latina como entre éstas y las universidades europeas. Tales redes son factores que contribuyen al trabajo en común entre instituciones de ambas regiones y de diversos países, por lo que favorecen los procesos de articulación y de convergencia en materia de educación superior. 
Una de ellas es Columbus (con sede en París), que es una asociación de 64 universidades europeas y latinoamericanas creada en 1987. Columbus promueve la constitución de redes de instituciones universitarias que actúan conjuntamente, y que ofrecen servicios de formación, de evaluación, de auditoria y de consultoría a universidades y a gobiernos. Su actual programa de trabajo incluye proyectos de convergencia de los sistemas de educación superior, de nuevas TIC, de internacionalización de la educación superior, de educación continua, de evaluación de las ingenierías, etc. (http://www.columbus-web.com.es).

La Asociación de Universidades del Grupo Montevideo fue creada en 1991, y está integrada por universidades públicas y privadas de Argentina, Brasil, Chile, Paraguay y Uruguay. Entre sus objetivos están: desarrollar conjuntamente una masa crítica de al to nivel entre sus miembros, promover la investigación científica y tecnológica y la formación continua, mejorar las estructuras de gestión de las universidades que la integran, y fomentar la interacción entre las instituciones miem-

bros. In su seno funcionan cinco cátedras UNESCO sobre cultura de la paz, sobre libertad de expresión, sobre derechos humanos, sobre nuevas técnicas de enseñanza e innovación pedagógica en la educación superior, y sobre microelectrónica. Por lo demás, en su ámbito funcionan diez comités académicos sobre distintos temas científico-tecnológicos, mientras en su página web (http://www. grupo montevideo.edu.uy) se informa sobre las actividades académicas desarrolladas desde el año 1991.

En 1948 se creó en Centroamérica el Consejo Superior Universitario Centroamericano ( CSUCA), organismo precursor de la integración subregional y del funcionamiento de redes interuniversitarias. En estas cinco décadas largas de existencia el CSUCA ha pasado por etapas muy importantes en cuanto a promover la integración universitaria en la región y en contribuir a su mejoramiento. Sus publicaciones y trabajos han sido muchas veces de gran interés tanto en Centroamérica como en el conjunto de América Latina. Hoy funciona dentro del marco del Sistema de Integración Centroamericano (SICA).

EI CSUCA está integrado por 16 universidades públicas de Centroamérica y Panamá (http://www.csuca.edu.gt). Por otra parte, creó el Sistema Centroamericano de Evaluación y Acreditación de la Educación Superior (SICEVAES), que, junto con el MEXA del MERCOSUR, constituyen los dos mecanismos subregionales de mayor significación en materia de aseguramiento y acreditación de la calidad de la educación superior en América Latina. 
EI CSUCA cuenta, además, con el Consejo Regional de Vida Estudiantil (CONREVE), y con una Red de Sistemas de Información Documental de Universidades Centroamericanas (Red SIID), que garantizan la interconexión telemática de los 16 sistemas de información documental de las universidades miembros. Tanto el SICEVAES como estos dos programas, contribuyen eficazmente a la convergencia y a la articulación de los sistemas universitarios de Centroamérica y Panamá.

En la década de los 70 se creó el Centro Interuniversitario de Desarrollo ( CINDA), con sede en Santiago de Chile. Está integrado por 28 universidades latinoamericanas y tres europeas (de España y de Italia). Su objetivo es el de articular esta red con el desarrollo regional y con el de cada uno de los países. Ha llevado a cabo numerosos estudios y trabajos de consultoría y de asesoramiento vinculados con el mejoramiento de la gestión y de la calidad en la educación superior (http:// www.cinda.cl).

En 1997 se constituyó en Buenos Aires la Red Latinoamericana de Cooperación Universitaria, integrada por universidades de 14 países. Dicha Red promueve la integración regional, la cooperación y la excelencia académica, así como la generación y la difusión del pensamiento en el área. Sus principales campos de trabajo están concentrados en la organización y gestión universitaria, en la movilidad y la homologación de estudios, en la acreditación institucional y por programas, y en las nuevas tecnologías para la educación superior.

Con objetivos similares se creó en 1993 la Asociación de Univensidades deAm érica Iatinay del Caribe para la Irtegración (Htp:/ / www .avlqpi.org), queestácompuestaporuniversidades de20países, y que prom ueve acciones de intercambio y de integración en $m$ ateria universitaria entresusm ienb ros con la consigna <La ComunidadIatinoam ericana de naciones es el nuevo lem ace la integración».

\section{HACIA LA CONSOLIDACIÓN DEL ESPACIO LATINOAMERICANO DE EDUCACIÓN SUPERIOR}

En los puntos anteriores hemos pasado revista a los trabajos, a los estudios y a las acciones llevadas a cabo por los países latinoamericanos, por sus universidades y por los organismos multilaterales para hacer frente a la fragmentación, y, en muchos casos, a la dispersión de los 
sistemas nacionales de educación superior, así como a la superposición de modelos y de tendencias en materia universitaria, sobre todo en las últimas décadas.

Se trataba de una situación similar -agudizada quizás por la trayectoria de siglos de los sistemas universitarios- a la que se enfrentaron los ministros de educación de 29 países europeos en 1999, en Bolonia, cuando decidieron poner en marcha el proceso de convergencia y de articulación denominado Proceso de B olonia. Los avances registrados en Europa en estos años son muy significativos, aunque las dificultades y los problemas sean también importantes. La transformación de los sistemas europeos de educación superior, históricamente diferenciados, hacia un modelo común, es un desafío que América Latina debe conocer y discutir. No para copiarlos ni para reproducirlos de manera mecánica, sino para partir de la experiencia europea de convergencia y de creación de su propio espacio común de educación superior.

Se podrá tomar o no la estructura bachillerato-maestría-doctorado, y se podrán asumir o no criterios análogos con el sistema europeo de créditos transferibles. Lo importante es trabajar para la articulación y la convergencia de los sistemas nacionales de educación superior con marcos y con criterios comunes. Quizás, en una primera etapa, en el contexto de las subregiones con proyectos comunes en ejecución: MERCOSUR y Centroamérica y Panamá. Luego, explorando la convergencia a nivel continental.

Es preciso realizar estudios y debates sobre la educación superior para buscar marcos y criterios comunes, con el fin de poder hacer frente a la actual fragmentación de los sistemas nacionales. Los aportes y las conclusiones del reciente Congreso Internacional América Latina y Europa ante los procesos de convergencia de la Educación Superior pueden ser muy útiles para esto.

Habría que pensar en la creación de organismos latinoamericanos del tipo de los europeos, como ENQA (European Net for Quality Assurance) o como J oint Qual ity Initiative para diseñar y para concertar criterios comunes en asuntos de calidad y de su aseguramiento. Esto, tanto con el fin de acreditar carreras, como, tal vez, de plantear acuerdos regionales acerca del reconocimiento, a niveles nacionales, de nuevas instituciones de educación superior que permitan hacer frente a la caótica situación actual en esta materia. Las experiencias recientes en 
tales procesos de países como Argentina, Chile y Colombia, pueden ser de mucha utilidad.

En cuestión de acreditación de carreras, los avances ya realizados en el MERCOSUR con el MEXA, en Centroamérica con el SICEVAES, y en México con su integración en el NAFTA, constituyen una base muy importante para construir un sistema que llegue a abarcar a toda América Latina. Por su parte, los trabajos del ProyectoALFA-ACRO también pueden ser de gran provecho para que el sistema latinoamericano de aseguramiento de la calidad se desarrolle con criterios convergentes y compatibles con los avances de los países europeos en el marco del Proceso de Bolonia. El funcionamiento de la RIACES constituye un antecedente interesante en esta tarea.

La consolidación del Espacio Latinoamericano de Educación Superior y su convergencia con el Europeo, requerirán un trabajo concertado y con proyectos en común entre los organismos multilaterales de cooperación y las redes interuniversitarias existentes. Esto será posible si se superan los criterios tradicionales de trabajo aislado - «cada uno en su chacra», como se dice en Argentina-, y se piensan estrategias, programas y proyectos conjuntos. Es un desafío difícil, pero hay que intentar superarlo.

La convergencia del Espacio Latinoamericano con el Europeo necesitará intensificar los trabajos en común entre organismos multilaterales, entre redes universitarias y entre instituciones de educación superior de América Latina y de Europa, para lo que el ProyectoALFAACRO constituye un antecedente significativo. Actualmente se está diseñando un proyecto de convergencia entre Columbus y CENEVAL de México - con participación de universidades de América Latina- denominado $6 \times 4$, por el que se propone desarrollar en seis carreras cuatro ejes comunes con los planteados por el Proceso de Bolonia.

Para el proceso de convergencia América Latina-Europa será muy importante el apoyo de organismos multilaterales, interuniversitarios y nacionales de cooperación europeos, en especial la Comisión Europea, a través de nuevos proyectos ALFA. La Comisión deberá tener un rol significativo en el desarrollo de esta idea estratégica de consolidar el Espacio Latinoamericano de Educación Superior en convergencia con el Europeo, de manera que se haga realidad el fortalecimiento del nuevo Espacio Común Unión Europea-América Latina de Educación Superior, creado por los J efes de Estado de ambas regiones. 


\section{BIBLIOGRAFÍA}

CONEAU (1997): Lineamientos para la evaluación institucional, Buenos Aires.

COMISIÓN EUROPEA: Proyecto «Acreditación y reconocimientos oficiales entre universidades del MERCOSUR y la Unión Europea» (ACRO), documentos varios.

DIAS SOBRINHO, J. (1995): «Avaliação institucional, instrumento da qualidade educativa», en Balzan, N. C. y Dias Sobrinho, J. (org.): Avaliação Institucional. teoria e experiências, São Paulo, Cortez Editora.

FERNÁNDEZ LAMARRA, N. (1999): Armonización de requerimientos educativos y migratorios entre países del MERCOSUR, Buenos Aires, olm, (mimeo).

- (2003): La educación superior argentina en debate. Situación, problemas y perspectivas, Buenos Aires, Eudeba-IESALC/UNESCO.

- (2003): «Los procesos de evaluación y acreditación universitaria en América Latina», en Evaluación de la Calidad y Acreditación, Madrid, UEALC, ANECA.

- (2004): «La necesidad de la convergencia de la Educación Superior en América Latina», en ponencia presentada en el Congreso Internacional «América Latina yEuropa ante los procesos de convergencia de la educación superior», B uenos Aires, Unión Europea-UNTREF (en prensa).

- (2004): «Una nueva agenda para la educación del futuro. La internacionalización de la educación virtual y la evaluación de su calidad», VIRTUAL EDUCA 2004 , Forum de Barcelona.

FERNÁNDEZ LAMARRA, N. y PULFER, D. (1992): Educación y recursos humanos en el mercosur. Armonización de políticas para la integración, Buenos Aires, INTAL/BID, (mimeo).

IESALC/UNESCO: Documentos en línea, http://www. iesalc.unesco.org.ve.

LEMAITRE, M. J . (2002): Aseguramiento de la calidad en tiempos de cambio: la experiencia de Chile, Cartagena de Indias, CNA.

LÉmEZ, R. (2002): La construcción yaplicación del Mecanismo de Acreditación MERCOSUR: fortalezas y debilidades, Proyecto ALFA-ACRO, Montevideo.

MORA, J -G. (2002): La evaluación y la acreditación de programas académicos en España y en la Unión Europea, Cartagena de Indias, CNA.

OEI: Documentación sobre programas y actividades, http://www.oei.org.es.

OUI: Documentación sobre programas, http://www.oui.iohe.qc.ca.

RISTOFF, D.: «Avaliação institucional: pensando princípios», en N. C. Balzan y Dias Sobrinho (org.), op. cit. 


\title{
Contactar
}

Revista lberoamericana de Educación

\author{
Principal OEI
}

